\title{
Computing Solution of the Flow due to a Nozzle
}

\author{
Bounab Noura \\ Laboratory of Pure and Applied \\ Mathematics LPAM \\ Department of Mathematics. \\ University of M'sila Algeria
}

\author{
Bouderah Brahim \\ Laboratory of Pure and Applied \\ Mathematics LPAM \\ Department of Computer Science. \\ University of M'sila Algeria
}

\begin{abstract}
The present work consider a bidimensional flow with a free surface of an incompressible and inviscid fluid due to a curved nozzle, where the effect of surface tension is not neglected. The computed accurate numerical solution with surface tension $T \neq 0$ via a serie truncation. However, there is a solution for each value of the Weber number $\alpha>5$
\end{abstract}

Keywords: Computation, Free surface, nozzle, Weber number

\section{INTRODUCTION}

Free surface flows have been studied by many authors F.Dias \& aL[1] B. Bouderah \& aL [2] [4] [9],, H. Mekias \& al [3] [5], Analytical data for flows issuing from horizontal nozzle far upstream are rather common literature, Gurevich [6]. Most of this results come from earlier work by Von Mises [7], F. Toison [8].

\section{NUMERICAL RESOLUTION}

\subsection{Problem Statement and Solution Method}

Let us consider a steady two-dimensional flow of an incompressible, irrotational and inviscid fluid issuing from a curved nozzle which forms an angle $\beta=\frac{\pi}{4}$ with the horizontal (see figure 1).

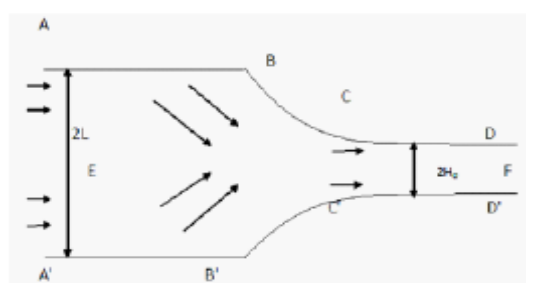

Fig:1 The $Z$ - plan.

One takes because of symmetry of the flow field,we need only consider the upper half of the flow region witch is between the $\mathrm{x}$-axis and the streamline $A B C D$ (Figure.2).

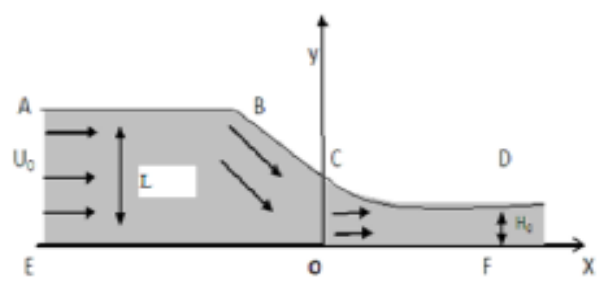

Fig 2: The upper half of the flow region $Z$-plan
We note that the flow is uniform and the velocity is equal to $\mathrm{U}$, and $\mathrm{L}$ as the unit length. We introduce the complex velocity potential $f=\varphi+i \psi$ without loss of generality, we choose $\psi=0$ on the streamline $A B C D, \varphi=0$, at $(x, y)=(0,1)$ and $\psi=-1$ on EOF. In order to use the conformal mapping techniques; the upper half of the flow region in the Z-plane will be mapped via the potential function $\mathrm{f}$ onto the semi -infinite strip $\{-\infty<\varphi<+\infty,-1<\psi<0\}$ (see Figure 3) .

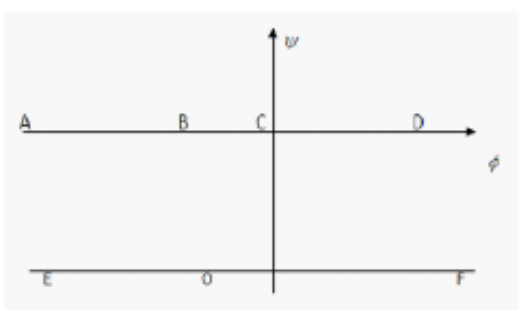

Fig 3 : The $f$ - Plan

We map it into the fourth quarter unit disk of the auxiliary $\omega$ plan by the transformation. (see Figure 5)

$$
f=\frac{2}{\pi} \ln \left(\frac{2 i \omega}{1+\omega^{2}}\right)
$$

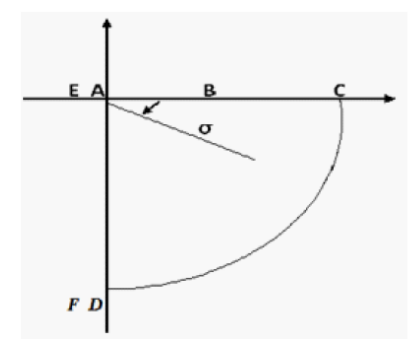

Fig 4 : The $w$ - Plan

On the free surface the pressure is constant and then the Bernoulli equation becomes

$$
q^{2}-\frac{2}{\alpha}\left(\frac{1}{R}\right)=1 \quad \text { on } C D
$$

Where $q$ is the flow speed, $R$ the radius of curvature of the free surface. The complex velocity rewrite in new variables $\tau$ and $\theta$ as $\xi=u-i v=\exp (\tau-i \theta)$. In dimensionless variables and using $\tau$ and $\theta$, (2) becomes

$$
\exp (2 \tau)+\frac{\pi}{\alpha} \tan (\sigma) \frac{\partial \theta}{\partial \varphi}=1 \quad \text {, on } C D
$$


Where $\alpha$ is the Weber number and $\omega=|\omega| \exp (i \sigma),-\frac{\pi}{2}<$ $\sigma<0$

Since $\xi$ must have singularity at two points correspond respectively at the point $\omega_{C}, \omega_{B}$ in the $\omega-$ plan, local behaviors of :

$\omega_{B}=b, \omega_{C}=1$ Are:

at $\omega_{B}=b: \xi \sim c_{1}\left(b^{2}-\omega^{2}\right)^{\frac{1}{2}} \quad$ as $\omega \rightarrow b$

with: $c_{1}=\left(\frac{3}{4}\right)^{\frac{1}{2}} \alpha\left(k_{1}\right)^{\frac{1}{2}}$

at $\omega_{C}=1: \xi \sim c_{2}\left(1-\omega^{2}\right)^{2-\frac{2 \gamma}{\pi}} \quad$ as $\omega \rightarrow 1$

with: $c_{2}=\left(\frac{\pi}{4}\right)^{1-\frac{\gamma}{\pi}} \alpha\left(k_{2}\right)^{1-\frac{\gamma}{\pi}}$

Following [1], [2] we seek $\xi(\omega)$ in the form :

$$
\begin{aligned}
\xi(\omega)=\left(b^{2}-\omega^{2}\right)^{\frac{1}{2}} & (1 \\
& \left.-\omega^{2}\right)^{2-\frac{2 \gamma}{\pi}} \exp \left(\sum_{k=1}^{\infty} a_{k} \omega^{2(k-1)}\right)
\end{aligned}
$$

The coefficients $a_{k}$ are to be found. The free surface CD is mapped by the transformation (1) into the fourth quarter unit circle. And we use the notation

$$
\omega=|\omega| \exp (i \sigma), \quad-\frac{\pi}{2}<\sigma<0
$$

In the relation (4), we obtain:

$$
\begin{array}{r}
\exp (\tau(\sigma))=(2 \cos \sigma)^{2-\frac{2}{\pi}} \exp \left(\sum_{k=1}^{\infty} a_{k} \cos (2(k-1) \sigma)\right) \\
\theta(\sigma)=-\left(3 \sigma-\frac{2 \gamma}{\pi} \sigma+\sum_{k=1}^{\infty} a_{k} \sin (2(k-1) \sigma)\right)
\end{array}
$$

\subsection{Computational Method}

Following Vanden-Broeck, Mekias and Bouderah ([1]- [4]), we solve the problem by truncating the infinite series in (4) after $\mathrm{N}$ terms. The $\mathrm{N}$ coefficients $a_{n}$ are found by collocation.

\subsubsection{Computation of the coefficients $\left(a_{k}\right)_{k=1, n}$ of} the series(4)

The initial data $\mathrm{A}^{(0)}$,

$\varepsilon ;$ kmax.

1. Computing: $A=\left(a_{i}\right)_{i=1, n}$

$$
\begin{gathered}
\left.E_{i j}^{(K)}=\left.\frac{\partial f_{i}(A)}{\partial a_{j}}\right|_{A=A^{(k)}}\right\} j=1, \ldots, n \\
f_{i}^{(k)}=1+\left(2 \cos \sigma_{i}\right)^{2-\frac{2}{\pi}} \exp \left(\sum_{k=1}^{\infty} a_{k} \cos \left(2(k-1) \sigma_{i}\right)\right) \\
+\frac{\pi}{\alpha} \tan \left(\sigma_{i}\right) * \\
i=1, \ldots, n
\end{gathered}
$$

2. Solve the linear system:

$$
\left.\sum_{k=1}^{n} E_{i j}^{(K)} \Delta A_{j}^{(k)}=f_{i}^{(k)}\right] i=1, \ldots, n
$$

\begin{tabular}{|c|c|c|c|}
\hline \multicolumn{4}{|c|}{ Table 1. Alpha $=10^{+8}$} \\
\hline$n$ & $a_{n}$ & $\mathrm{n}$ & $a_{n}$ \\
\hline 1 & $4.954010^{-9}$ & 26 & $-3.459810^{-12}$ \\
\hline 2 & $-3.303810^{-9}$ & 27 & $-3.144810^{-12}$ \\
\hline 3 & $-6.587810^{-10}$ & 28 & $-2.874910^{-12}$ \\
\hline 4 & $-2.828010^{-10}$ & 29 & $-2.620510^{-12}$ \\
\hline 5 & $-1.566010^{-10}$ & 30 & $-2.400810^{-12}$ \\
\hline 6 & $-9.971610^{-11}$ & 31 & $-2.191310^{-12}$ \\
\hline 7 & $-6.876410^{-11}$ & 32 & $-2.008810^{-12}$ \\
\hline 8 & $-5.041910^{-11}$ & 33 & $-1.833310^{-12}$ \\
\hline 9 & $-3.837810^{-11}$ & 34 & $-1.678910^{-12}$ \\
\hline 10 & $-3.026910^{-11}$ & 35 & $-1.529110^{-12}$ \\
\hline 11 & $-2.437110^{-11}$ & 36 & $-1.396010^{-12}$ \\
\hline 12 & $-2.009710^{-11}$ & 37 & $-1.265910^{-12}$ \\
\hline 13 & $-1.677610^{-11}$ & 38 & $-1.148910^{-12}$ \\
\hline 14 & $-1.425310^{-11}$ & 39 & $-1.033710^{-12}$ \\
\hline 15 & $-1.219810^{-11}$ & 40 & $-9.285010^{-13}$ \\
\hline 16 & $-1.058510^{-11}$ & 41 & $-8.238610^{-13}$ \\
\hline 17 & $-9.225210^{-12}$ & 42 & $-7.261010^{-13}$ \\
\hline 18 & $-8.131610^{-12}$ & 43 & $-6.269710^{-13}$ \\
\hline 19 & $-7.183410^{-12}$ & 44 & $-5.298710^{-13}$ \\
\hline 20 & $-6.407210^{-12}$ & 45 & $-4.247810^{-13}$ \\
\hline 21 & $-5.719010^{-12}$ & 46 & $-3.042610^{-13}$ \\
\hline 22 & $-5.147310^{-12}$ & 47 & $-1.277410^{-13}$ \\
\hline 23 & $-4.630810^{-12}$ & 48 & $2.712210^{-13}$ \\
\hline 24 & $-4.196710^{-12}$ & 49 & $2.867110^{-12}$ \\
\hline 25 & $-3.798110^{-12}$ & 50 & $-9.244510^{-11}$ \\
\hline
\end{tabular}

3. Computing:

$$
\left.A_{i}^{(k+1)}=A_{i}^{(k)}+\Delta A_{i}^{(k)}\right] i=1,2, \ldots, n
$$

4. If :

$$
\left.\begin{array}{c}
\left.\left|f_{i}\left(A^{(k+1)}\right)\right|<\in\right] i=1,2, \ldots, n \\
k>k \max \\
\text { is verified }
\end{array}\right]
$$

\section{Stop}

Remark: using the algorithm of Jordan with total pivotation implied.

Some coefficients $\left(a_{k}\right)$ of the series (4) for different values of number of weber Alpha 


\begin{tabular}{|c|c|c|c|}
\hline \multicolumn{6}{|c|}{ Table 2. Alpha=100 } \\
\hline $\mathrm{n}$ & $\mathrm{a}_{n}$ & $\mathrm{n}$ & $\mathrm{a}_{n}$ \\
\hline 1 & $4.954010^{-3}$ & 26 & $-3.366910^{-6}$ \\
2 & $-3.303810^{-3}$ & 27 & $-3.063310^{-6}$ \\
3 & $-3.587810^{-4}$ & 28 & $-2.796410^{-6}$ \\
4 & $-2.794410^{-4}$ & 29 & $-2.558010^{-6}$ \\
5 & $-1.546910^{-4}$ & 30 & $-2.347910^{-6}$ \\
6 & $-9.810010^{-5}$ & 31 & $-2.130410^{-6}$ \\
7 & $-6.768310^{-5}$ & 32 & $-1.993910^{-6}$ \\
8 & $-4.946210^{-5}$ & 33 & $-1.711910^{-6}$ \\
9 & $-3.768110^{-5}$ & 34 & $-1.747010^{-6}$ \\
10 & $-2.962810^{-5}$ & 35 & $-1.377110^{-6}$ \\
11 & $-2.388510^{-5}$ & 36 & $-1.486710^{-6}$ \\
12 & $-1.964510^{-5}$ & 37 & $-1.197010^{-6}$ \\
13 & $-1.640910^{-5}$ & 38 & $-1.049910^{-6}$ \\
14 & $-1.390510^{-5}$ & 39 & $-1.141310^{-6}$ \\
15 & $-1.193410^{-5}$ & 40 & $-9.007310^{-7}$ \\
16 & $-1.030910^{-5}$ & 41 & $-7.224410^{-7}$ \\
17 & $-9.018210^{-6}$ & 42 & $-7.970710^{-7}$ \\
18 & $-7.927310^{-6}$ & 43 & $-6.998110^{-7}$ \\
19 & $-7.004310^{-6}$ & 44 & $-4.708410^{-7}$ \\
20 & $-6.244510^{-6}$ & 45 & $-4.350410^{-7}$ \\
21 & $-5.570010^{-6}$ & 46 & $-4.736510^{-7}$ \\
22 & $-5.013410^{-6}$ & 47 & $-3.415510^{-7}$ \\
23 & $-4.515010^{-6}$ & 48 & $-1.562310^{-7}$ \\
24 & $-4.090810^{-6}$ & 49 & $-1.259510^{-7}$ \\
25 & $-3.706210^{-6}$ & 50 & $-8.264810^{-6}$ \\
\hline \multicolumn{5}{|c}{} & \\
\hline
\end{tabular}

\subsubsection{Calculate of the free surface}

On the interval $\left[\frac{-\pi}{2}, 0\right]$, we introduce $(\mathrm{N}+1)$ on mesh points,

$$
\sigma_{I}=\frac{\pi}{2(N+1)}\left(I-\frac{1}{2}\right), \quad I=1, \ldots \ldots \ldots+1
$$

We obtain $(\mathrm{N}+1)$ nonlinear algebraic equations of $(\mathrm{N}+1)$ unknowns $a_{k}$, the shape of the free surface is determined by integrating numerically the relation :

$$
\begin{aligned}
\frac{\partial x}{\partial \sigma}=\frac{2}{\pi} \cot \sigma_{I}\left(2 \cos \sigma_{I}\right)^{2-\frac{2 \gamma}{\pi}} \exp \left(\sum_{k=1}^{N} a_{k} \cos \left(2(k-1) \sigma_{I}\right)\right) \\
\times \cos \left(3 \sigma-\frac{2 \gamma}{\pi} \sigma_{I}\right. \\
\left.+\sum_{k=1}^{N} a_{k} \sin \left(2(k-1) \sigma_{I}\right)\right)
\end{aligned}
$$

$$
\begin{aligned}
\frac{\partial y}{\partial \sigma}=\frac{2}{\pi} \cot \sigma_{I}\left(2 \cos \sigma_{I}\right)^{2-\frac{2 \gamma}{\pi}} \exp \left(\sum_{k=1}^{N} a_{k} \cos \left(2(k-1) \sigma_{I}\right)\right) \\
\times \sin \left(3 \sigma-\frac{2 \gamma}{\pi} \sigma_{I}\right. \\
\left.+\sum_{k=1}^{N} a_{k} \sin \left(2(k-1) \sigma_{I}\right)\right)
\end{aligned}
$$

The Euler's method was sufficient to solve numerically the relation above.

\section{DISCUSSION AND REPRESENTATION OF RESULTS}

\subsection{Solution without Surface Tension}

$$
(\alpha \rightarrow \infty, g=0)
$$

When the effect of surface tension is neglected in the free surface condition [9], the number of Weber $\alpha \rightarrow \infty$, the equation

(4) becomes :

$$
\exp (2 \tau)=v_{x}^{2}+v_{y}^{2}=1, \quad \text { on the free surface }
$$

Where the forces of gravity are not taken account; the exact and analytical solution is find via free stream line theory of Kirchhoff. Also, a numerical solutions were computed using the procedure described above for $\beta=\left(\frac{\pi}{4}\right)$, the solution is wrote like : $\xi(\omega)=\omega$, it's the same obtained by Khrichoff (Batchelor 1967).

In this case, the numerical computational shows that there is a critical value $\alpha_{\max }=1000, \alpha>\alpha_{\max }$, when $\alpha>\alpha_{\max }$, within the $\varepsilon \approx 10^{-8}$ error. We show in this case that the free surface flow for the Weber number $\alpha=10^{+8}$ and the exact solution find analytically are the same. (see figure 5).

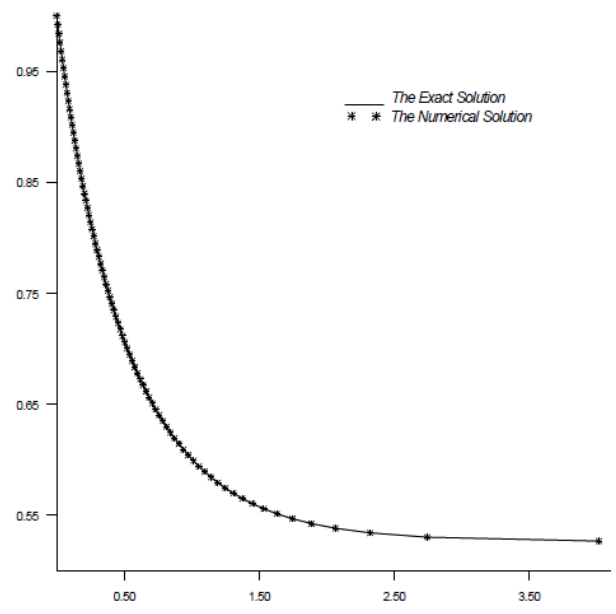

Fig 5. The exact and numerical solutions

\subsection{Surface Tension Effect $T \neq 0, g=0$}

When the surface tension is included in the free surface conditions, the numerical procedure shows that there exists a solution for various $\alpha \geq \alpha^{*}=5$ for the angle $\beta=\frac{\pi}{4}$. As $\mathrm{n}$ increase the coefficients $a_{n}$ decrease rapidly, it is also noted that the series

$$
\sum_{k=1}^{+\infty} a_{k} t^{2 k}
$$

Is absolutely convergent because we have:

$$
\sum_{k=1}^{+\infty}\left|a_{k} t^{2 k}\right| \leq \sum_{k=1}^{+\infty}\left|a_{k}\right| \leq \sum_{k=1}^{+\infty}\left(\frac{5}{6}\right)^{k} .
$$

(see figure 6-10). 


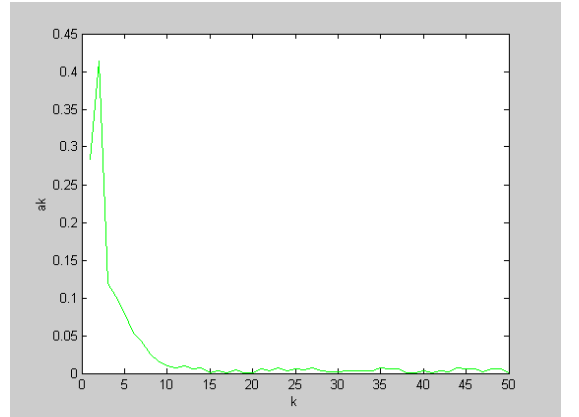

Fig 6: The coefficients $\left|a_{k}\right|$ for $\alpha=5.5$

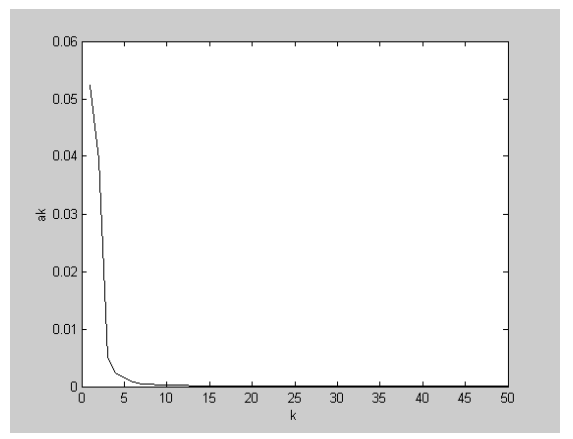

Fig 7: The coefficients $\left|a_{k}\right|$ for $\alpha=10$

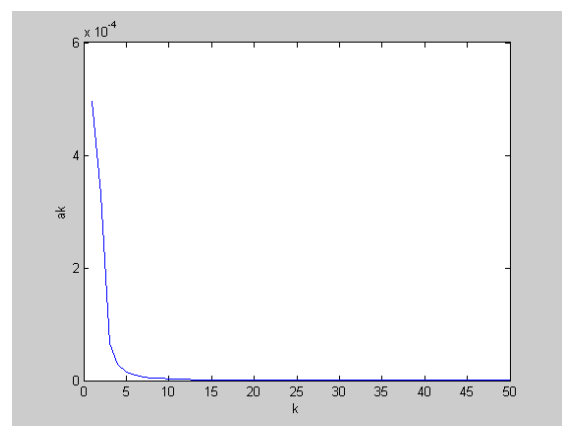

Fig 8: The coefficients $\left|a_{k}\right|$ for $\alpha=10^{3}$

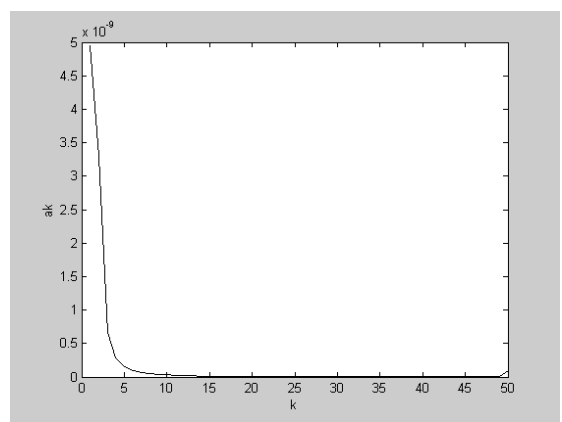

Fig 9: The coefficients $\left|a_{k}\right|$ for $\alpha=10^{8}$

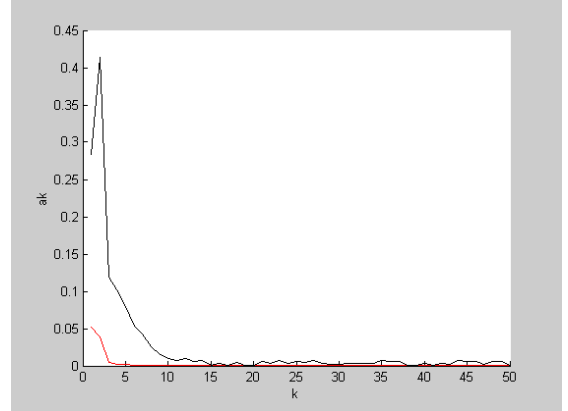

Fig 10: Comparison between the coefficients $\left|a_{k}\right|$ for $\alpha=10$ and $\alpha=5.5$

The numerical scheme ceases to converge for all the values of the parameter $\alpha \leq \alpha^{*}$. It is noted that the convergence is faster when $\alpha \rightarrow \infty$ and it is slower when $\alpha \rightarrow \alpha^{*}=5$. Finally, the form of the free surface is represented for various values of the Weber number $\alpha$ (See Figure.11.)

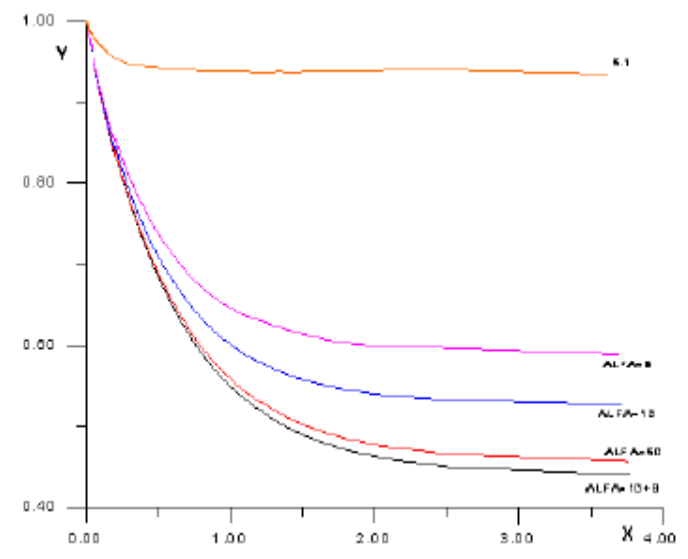

Fig 11 : The free surface flow for various values of the Weber number $\alpha$

\section{CONCLUSION}

In a forthcoming work, and after some research studies in this domain, we raise the interesting other work problem by adopting the method of the finite volume technique, to find the approach solution.

\section{REFERENCES}

[1] F.Dias and J.M. Vanden-Broeck,"Flows emerging from a nozzle and falling under gravity ,"J.Fluid Mech .213, 465(1990)

[2] B. Bouderah and H. Mekias, A cybernetic approach to the problem of cusp free-surface flow caused by a line sink on a sloping bottom, Kybernetes 31, ํ2,(2002),305-316

[3] H. Mekias , J. M. Vanden-Broeck,"Supercritical freesurface flow with a stagnation point due to a submerged source", Phys. Fluids A, 1, №10,(1989), 1694

[4] B. Bouderah, H. Mekias,"The effects of surface tension on a two dimensional free surface flow produced by a source", Far East Journal of Applied Mathematics, 4, (2000),159 170 
[5] A.Gasmi and H.Mekias, "The effect of surface tension on the contraction coefficient of a jet emanating ," J. Phys. A:Math. Gen 36 p. 851-862 (2003).

[6] M.I.Gurevich,"Theory of Jets in Ideal Fluids."(Pergamon, New York, 1966)

[7] R. Von Mises, "Berechnung von Ausfluss and Uberfallzahlen," Zeitschrift des Vereines Deutscher Ingenieure 6121 (1917)
[8] F. Toison, J. Hureau,"potential flow issuing from a twodimensional curved nozzle," Phys. of fluids . Volume 13,Number3 . (March 2001)

[9] B. Bouderah, H. Serguine and A. gasmi,"Zero Gravity of Free-Surface Jet Flow", International Mathematical Forum, 2,2007 , no. 66, 3273 - 3277 